ARTICLE

\title{
Regioselective activation of benzocyclobutenones and dienamides lead to anti-Bredt bridged-ring systems by a [4+4] cycloaddition
}

\author{
Jianyu Zhang ${ }^{1}$, Xi Wang $^{1} \&$ Tao Xu (i) ${ }^{1,2}$ 凶
}

To the best of our knowledge, bridgehead carbon benzofused-bridged ring systems have previously not been accessible to the synthetic community. Here, we describe a formal type-II $[4+4]$ cycloaddition approach that provides fully $\mathrm{sp}^{2}$-carbon embedded anti-Bredt bicyclo [5.3.1] skeletons through the Rh-catalyzed $\mathrm{C}_{1}-\mathrm{C}_{8}$ activation of benzocyclobutenones (BCBs) and their coupling with pedant dienamides. Variously substituted dienamides have been coupled with BCBs to provide a range of complex bicyclo[5.3.1] scaffolds (>20 examples, up to $89 \%$ yield). The bridged rings were further converted to polyfused hydroquinolinecontaining tetracycles via a serendipitously discovered transannular 1,5-hydride shift/Prinslike cyclization/Schmidt rearrangement cascade.

\footnotetext{
${ }^{1}$ Molecular Synthesis Center and Key Laboratory of Marine Drugs, Ministry of Education, School of Medicine and Pharmacy, Ocean University of China, Qingdao, China. ${ }^{2}$ Laboratory for Marine Drugs and Bioproducts and Open Studio for Druggability Research of Marine Natural Products, Pilot National Laboratory for Marine Science and Technology, Qingdao, China. ${ }_{\text {email: xutao@ouc.edu.cn }}$
} 
D ridged ring systems are key structural motifs in many important functional molecules ${ }^{1-3}$. The skeleton were characterized by its size (e.g., bicyclo[m.n.1]) and ring strain (hybridization form of the bridgehead carbon (BC)). While the $\mathrm{sp}^{3}$-hybridized $\mathrm{BC}$ are commonly seen in many natural products, the $\mathrm{sp}^{2}$-hybridized $\mathrm{BC}$ underpins certain limitation on both the ring size $(m+n \geq 7)$ as well as ring strain ${ }^{3}$. However, consecutive $\mathrm{sp}^{2}$-hybridized carbon centers and/or bridgehead benzofused [m.n.1] systems are unusual $(m+n>8)$ and, to the best of our knowledge, unknown to chemist when it was confined in a small ring $(m+n \leq 8)$ framework (Fig. 1a $)^{4,5}$. We are herein set off to challenge these limitations. Bicyclo[5.3.1] bridged ring systems with anti-Bredt olefins at the bridgehead position are widely found in bioactive natural products (Fig. 1b) ${ }^{6-8}$, whose total synthesis was highly dictated by the efficiency of assembling such scaffolds ${ }^{9,10}$. The state-of-art cycloaddition methods in constructing anti-Bredt bicyclo[5.3.1] scaffolds ${ }^{11-14}$ are two: (1) type-II intramolecular Diels-Alder reactions developed by Shea et al. ${ }^{15,16}$, which requires activating substituent on dienophile, otherwise the reactions are mostly in gas phase; (2) a seminal report by Wender et al. ${ }^{17,18}$ using Ni-catalyzed type-II $[4+4]$ intramolecular cycloaddition under diluted conditions between 1,3-dienes (Fig. 1c) ${ }^{19}$. A general, catalytic and diversifiable cycloaddition strategy remained elusive, especially for the highly strained anti-Bredt ${ }^{20,21}$ bridged skeletons. The transition metalcatalyzed intramolecular $[4+2]$ annulation via $\mathrm{C}_{1}-\mathrm{C}_{2}$ bond activation $^{22-33}$ of benzocyclobutenones (BCBs) has emerged as an attractive approach for preparing fused-and bridged ring systems pioneered by Liebeskind ${ }^{34}$ and Dong ${ }^{35,36}$ (Fig. 1d). However, no catalytic $[4+4]$ annulation via oxidative $\mathrm{C}-\mathrm{C}$ activation has been known, not to mention forming the highly strained anti-Bredt bridged ring systems ${ }^{37}$. Accessing anti-Bredt bridged bicycles remained elusive since only a few examples had been known starting from cyclobutanones ${ }^{38-43}$. To the best of our knowledge, no report had been known to construct bridged ring systems that bears highly strained consecutive double bond surrounding the BC.

Herein, we disclose our discovery of a type-II $[4+4]$ annulation between BCBs and dienamides enabled by $\mathrm{Rh}$-catalyzed regioselective $C_{1}-C_{8}$ bond activation (Fig. 1e).

\section{Results and discussion}

The challenges are three-fold: (1) there is no precedent report of benzo-fused $\mathrm{BC}$ in bicyclo[5.3.1] systems; (2) the kinetically $\mathrm{C}_{1}-\mathrm{C}_{8}$ rhodaindanone would be readily isomerize to its thermodynamically stable $\mathrm{C}_{1}-\mathrm{C}_{2}$ cleaved counterpart ${ }^{44}$; (3) competitive fused ring formation through normal cut and sew pathway. Our investigation commenced with preparation of 1a through amide formation in $72 \%$ yield between our previously reported 3NHMe BCB and the known dienoic acid (see supporting information (SI) for detail $)^{45}$. We tested our hypothesis by using 2.5 $\mathrm{mol} \%$ of $[\mathrm{Rh}(\mathrm{nbd}) \mathrm{Cl}]_{2}$ and $12 \mathrm{~mol} \%$ of DPPP, unfortunately, only the fused tricycle $\mathbf{2} \mathbf{a}^{\prime}$ was isolated in $45 \%$ yield, presumably from the "cut and sew" process followed by a double bond migration (entry 1, Fig. 2). It is noted that the type-I $[4+4]$ benzofused ring $^{46,47}$ product was not observed. When switching to other bidetate ligand with varied bite-angles, namely $(S)-\mathrm{H}_{8}$-BINAP and $(S, S)$-DIOP. Surprisingly, the desired anti-Bredt benzofused aza-bicyclo[5.3.1] bridged product $2 \mathbf{2 a}$ was successfully isolated ( $45 \%$ and $40 \%$ yield, respectively) together with the side product $2 \mathbf{a}^{\prime}$ (38\% and $47 \%$ yield, respectively, entry 2 and 3). In both

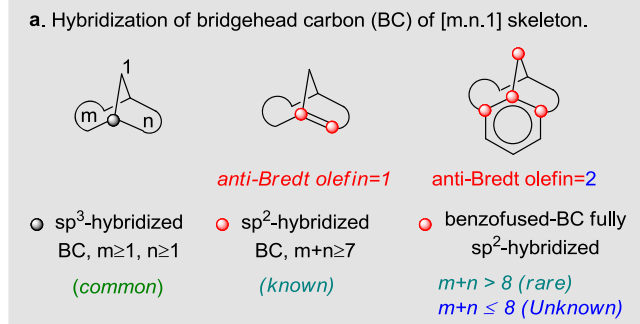

c. State-of-art cycloaddition approach for anti-Bredt bicyclo[5.3.1] ring

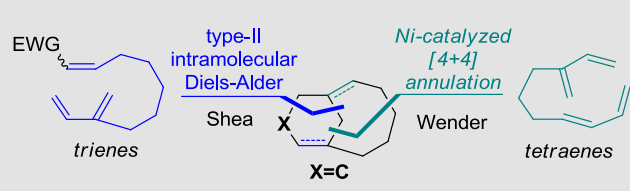

e. Our design: Bridged-ring formation via [4+4] annulation from $B C B$
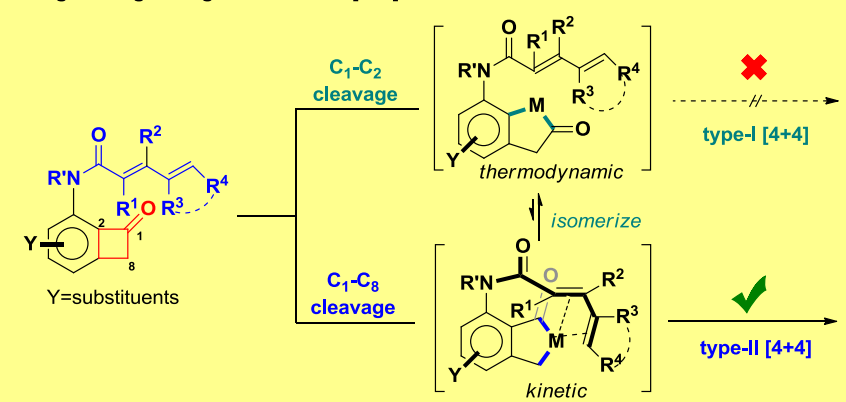

kinetic b. Representative NPs containing anti-Bredt bicyclo[5.3.1] scaffolds

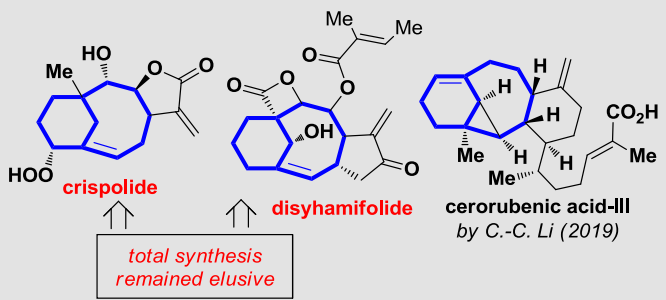

d. "Cut \& Sew" type [4+2] using BCBs and CBs pioneered by Dong etc.

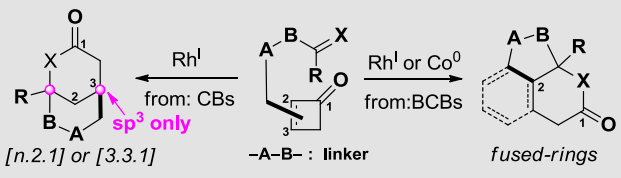

Fig. 1 Representative natural products and design of a [4+4] annulation approach to access anti-Bredt bicyclo[5.3.1] skeleton. a Hybridization of bridgehead carbon of [m.n.1] skeleton. b Representative NPs containing anti-Bredt bicyclo[5.3.1] scaffolds. c State-of-art cycloaddition approaches for antiBredt bicyclo[5.3.1] rings. d "Cut and sew" type [4+2] using BCBs and CBs pioneered by Dong, etc. e Our design: bridged ring formation via [4 + 4] annulation from BCBs. 


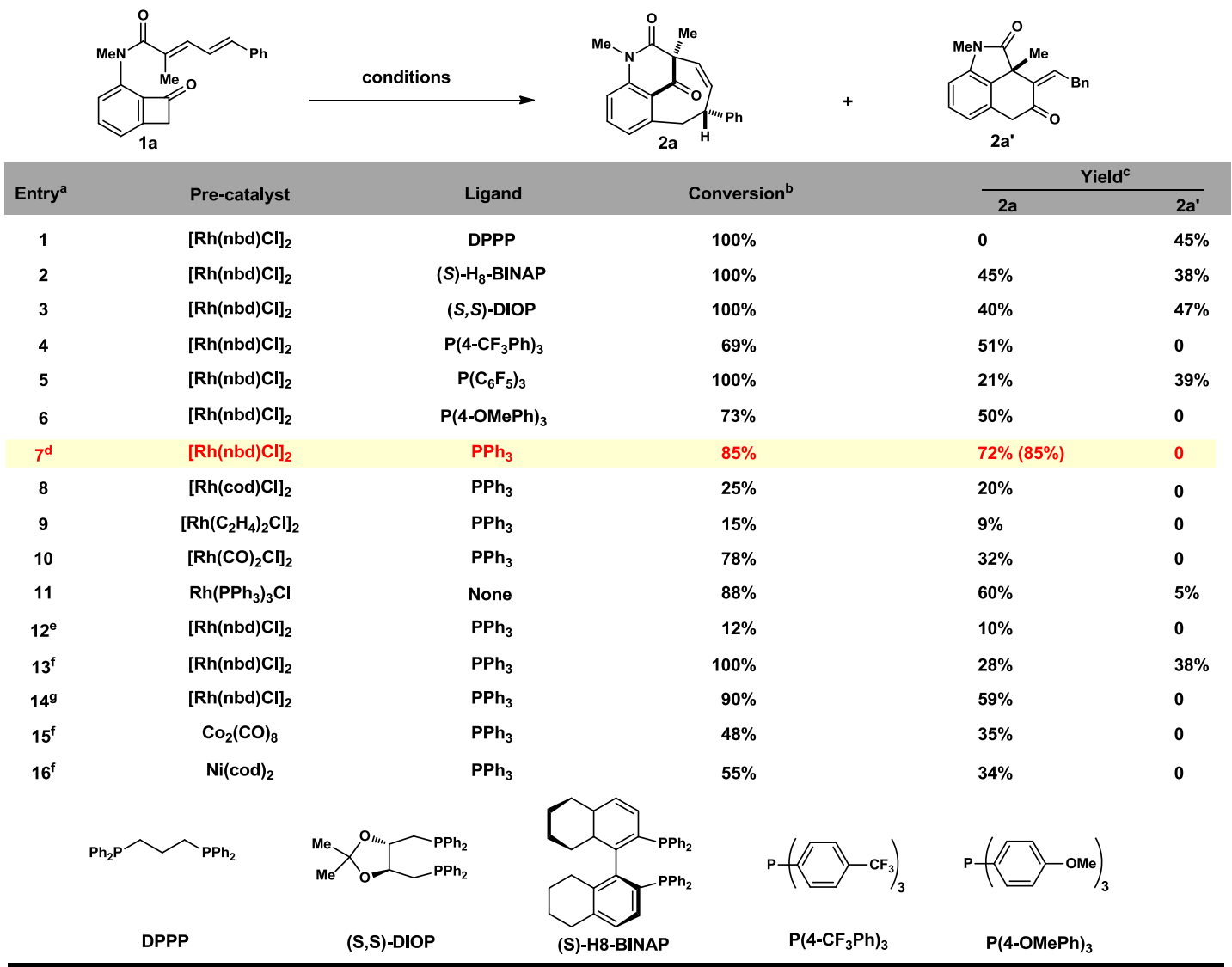

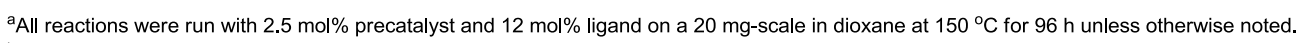

${ }^{b}$ Conversions were determined based on recycled $1 \mathrm{a}$.

Isolated yield;

${ }^{\mathrm{d}}$ Numbers in parenthesis are brsm yield;

${ }^{\mathrm{e}}$ The reaction was run at $110^{\circ} \mathrm{C}$;

${ }^{\mathrm{f}} 5 \mathrm{~mol} \%$ of precatalyst and $6 \mathrm{~mol} \%$ of $\mathrm{PPh}_{3}$ were used;

${ }^{9}$ Toluene was used as solvent.

Fig. 2 Condition optimizations. Precatalysts, ligands, temperatures and their ratios have been screened.

cases, product $2 \mathrm{a}$ 's ee values were 0 . These results led us to speculate that only one phosphine-center served as dative ligand based on the 18d-electron rule. Mono-dentate $\mathrm{P}\left(4-\mathrm{CF}_{3} \mathrm{Ph}\right)_{3}$ greatly improved the selectivity yielding only $2 \mathrm{a}$ in $51 \%$ yield (entry 4). However, the analogous $\mathrm{P}\left(\mathrm{C}_{6} \mathrm{~F}_{5}\right)_{3}$ only lead to significantly decreased yield of $\mathbf{2 a}(21 \%)$ and selectivity (39\% yield of $2 \mathbf{a}^{\prime}$ ), presumably due to the loose coordinating ability owing to its $\pi$-acidity as well as the large cone angle (entry 5$)^{48}$. The electronrich $\mathrm{P}(4-\mathrm{OMePh})_{3}$ provided elevated yield $(50 \%)$ of $\mathbf{2 a}$ albeit with $73 \%$ conversion (entry 6). Surprisingly, it was found that the feedstock $\mathrm{PPh}_{3}$ provide the highest $72 \%$ isolated yield based on $85 \%$ conversion while preserving the complete selectivity for the bridged products (entry 7, with light yellow background). Encouraged by this result, a series of pre-catalysts were screened, but the yields of 2a ranged between 9 and 32\% (entry 8-10). The Wilkinson catalyst was also used but proved less chemoselective as both $\mathbf{2 a}$ and $\mathbf{2} \mathbf{a}^{\prime}$ were obtained (entry 11). An attempt to lower the reaction temperature from 150 to $110^{\circ} \mathrm{C}$ proved unfruitful, as 2a was only obtained in $10 \%$ yield with most of 1a recovered (entry 12). The $\mathrm{Rh} /$ ligand ratio was also proved crucial to the success of this $[4+4]$ annulation pathway, since decreased efficiency ( $28 \%$ yield of $\mathbf{2 a}$ ) and loss of selectivity ( $38 \%$ yield of $\mathbf{3 a}$ ) was observed when lowering the loading of $\mathrm{PPh}_{3}$ (entry 13). Toluene is an inferior solvent (entry 14). First row late transition metal are known to cleave $\mathrm{C}_{1}-\mathrm{C}_{8}$ bond of $\mathrm{BCB}$, but $\mathrm{Co}^{0}$ and $\mathrm{Ni}^{0}$ complex were tested and proved less efficient (entry 15-16).
With optimal condition in hand, we set off to explore the reaction scope (Fig. 3). A broad series of dienamide coupled BCB substrates with different steric and electronic properties have been examined. Delightedly, good to high yields of bicyclo[5.3.1] bridged rings were obtained. It was found that changing the electronegativity of aryl substituents on $\delta$-position of dienamides (1a-1d) does not affect the efficacy, and $68-75 \%$ yields were obtained, with a slight trend for para-substituted effect favoring electron-withdrawing groups (entry 1-4, Fig. 3). The alkyl substituents at $\alpha$ and $\beta$-position of dienamide were also investigated (entry 5-8). It was gratifyingly to find that ethyl (1e), cyclopropylmethyl (1f) as well as hydrogen (1g-h) at $\alpha$-position were all well tolerated yielding desired bridged products $\mathbf{2 e - h}$ in useful yields (46-65\%). It is noteworthy that no cleavage of cyclopropyl moiety was detected, revealing good chemoselectivity of this catalyst system. Variation of $\mathrm{R}$ group on the nitrogen promised potential use in complex settings, as $89 \%$ yield of $2 \mathbf{i}$ was isolated (95\% brsm), when Bn (1i) was used (entry 9). Increasing the steric bulkiness to cyclopropylmethyl (1j) resulted in no less efficiency ( $79 \%$ yield of $\mathbf{2 j}$, entry 10 ). The reaction demonstrated excellent chemoselectivity when $\mathbf{1 k}$ was employed as substrate, only bridged $[4+4]$ product $\mathbf{2 k}$ ( $79 \%$ yield, $94 \%$ brsm) was observed but no traditional $[4+2]$ product (entry 11). The robustness toward electronic variation on $\mathrm{BCB}$ was enlightened by obtaining 21 in $66 \%$ yield ( $81 \%$ brsm, entry 12 ). A grand challenge in "migratory insertion" is the unviability of sterically 


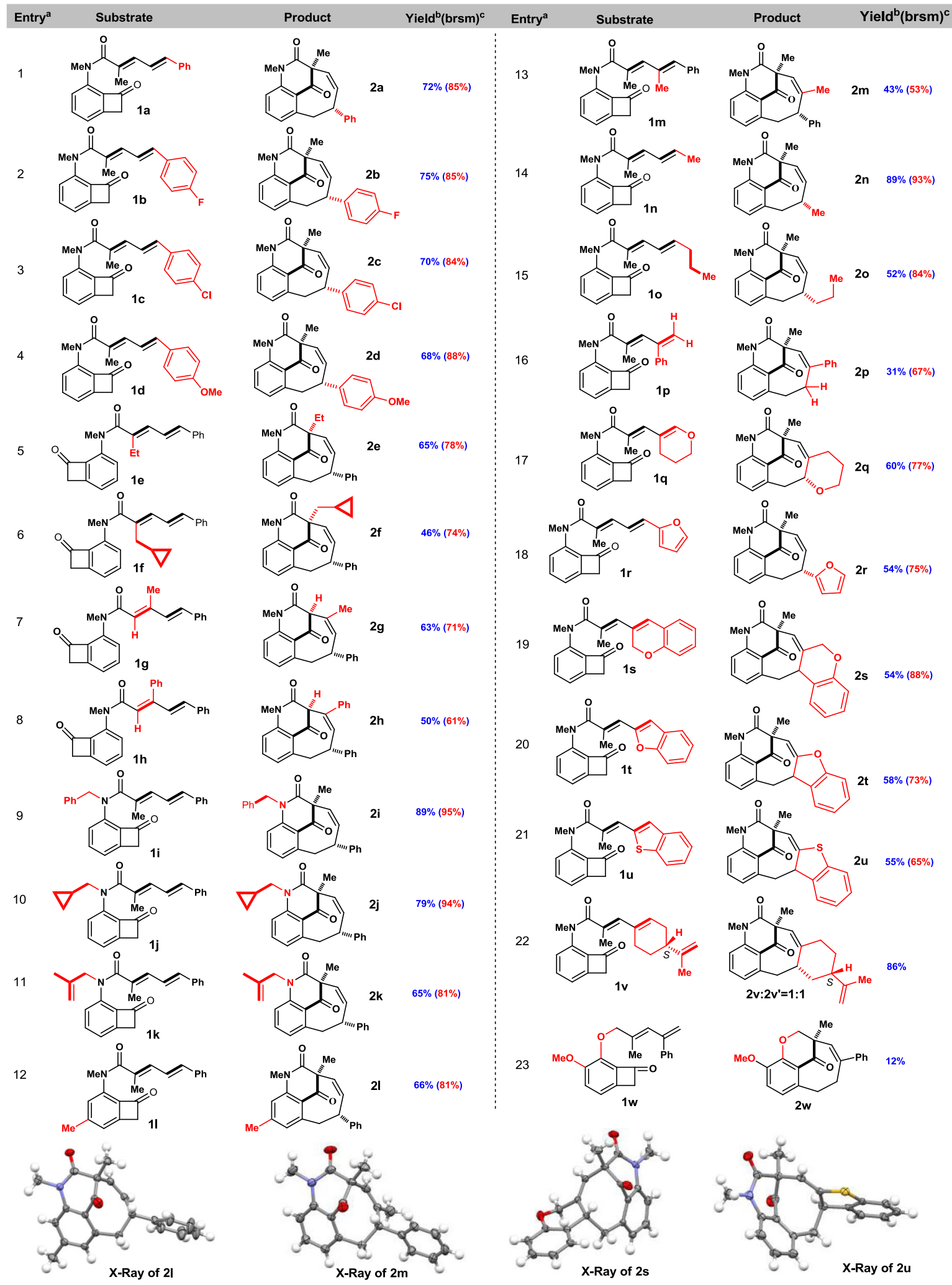

Fig. 3 Substrate scope. a Conditions: all reactions were run with $2.5 \mathrm{~mol} \%$ rhodium complex and $12 \mathrm{~mol} \%$ ligand in dioxane at $150{ }^{\circ} \mathrm{C}$ for $96 \mathrm{~h}$ unless

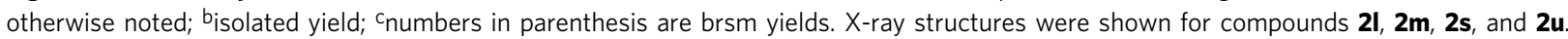

demanding multi-substituted olefins as coupling partners, presumably due to their low binding affinity. We postulated that dienamides are potential chelating ligands, which increased their binding ability to the rhodaindanone complex. The tetrasubstituted dienamide $\mathbf{1} \mathbf{m}$ was synthesized and the resulting $[4+4]$ annulation product $\mathbf{2 m}(84 \%$ brsm yield) was undoubtedly verified through X-ray crystallography together with the aforementioned 21 (entry 12 and 13). A major competitive side 
reaction after migratory insertion is the tendency to undergo $\beta-\mathrm{H}$ elimination when a $\beta-\mathrm{H}$ is available. Gratifyingly, in our trials to effect the alkyl substituted dienamides $\mathbf{1 n}$ and $\mathbf{l o}$ to the benzofused bridged skeletons, high to moderate yields were obtained, respectively, for 2 n ( $89 \%$ yield) and $2 \mathbf{o}$ (52\% yield) as the only isolated product in each case (entry 14 and 15). The terminal dienamide 1p underwent a certain decomposition which cause isolation of desired $2 \mathbf{p}$ in $31 \%$ yield albeit with moderate $67 \%$ brsm yield (entry 16). When the electronic property of dienamide was altered (1q), the desired $[4+4]$ annulation took place without loss of efficacy, obtaining $2 \mathbf{q}$ in $60 \%$ yield (entry 17). It was very encouraging to find that extended conjugated tetraenamide $1 \mathbf{r}$ react smoothly yielding $2 \mathbf{r}$ in $54 \%$ yield and the electron-rich furan moiety was well preserved (entry 18). When using even challenging (possessing cis-terminal olefins) cyclic dienamides $1 \mathbf{s}-\mathbf{u}$, the desired $[4+4]$ annulation reactions proceeded without any difficulties, affording $\mathbf{2 s} \sim \mathbf{u}$ in synthetically useful yields (54-58\%, entry 19-21). The product $2 \mathbf{t}$ and $2 \mathbf{u}$ suggested that aromaticity can be deprived, since the X-ray crystallographic experiment undisputedly showed an exo-olefin for product $\mathbf{2 u}$ even with the possibility for re-aromatization (Fig. 3, bottom). This might be caused by the highly strained antiBredt aza-bicyclo[5.3.1] skeleton. An effort to generate enantioselective bridged rings through chiral substrate induction was carried out using $1 \mathbf{v}$, delightedly, the resulting product $2 \mathbf{v}$ and $2 \mathbf{v}^{\prime}$ was obtained in a 1:1 ratio as inseparable isomers in a combined $86 \%$ yield (entry 22 ). These provide valuable clues in our future endeavor toward enantioselective $[4+4]$ annulation study. Additionally, we also found the ether substrate $\mathbf{1 w}$ yielded desired $[4+4]$ product $2 \mathrm{w}$, albeit in $12 \%$ yield. This indicated that our [4 +4 ] conditions can be extrapolated to O linked diene substrate albeit in low yield (entry 23).

With the highly diastereomerically pure benzo-fused bridged cyclic compounds $\mathbf{2}$ in hand, we hypothesized that if a Schmidt rearrangement reaction can be performed (Fig. 4), which would further extended the structural diversity ${ }^{49-53}$ based on this $[4+$ 4] annulation strategy. In addition, it may be considered as a reaction surrogate for using the oxindole 3 as the annulation substrate, which has been an elusive substrate in $\mathrm{C}-\mathrm{C}$ bond activation due to the more reactive amide bonds.

After extensive investigations, we were delighted to discover that the bicyclo[5.3.1] bridged tricycles can readily undergo a clean rearrangement reaction with isolated yields ranging from 51 to $62 \%$ yield (Fig. 5). A careful cultivation of a single crystal of the product $5 \mathrm{~g} / 5 \mathrm{n}$ and X-ray crystallographic analysis revealed that a densely functionalized and polyfused ring systems were obtained.

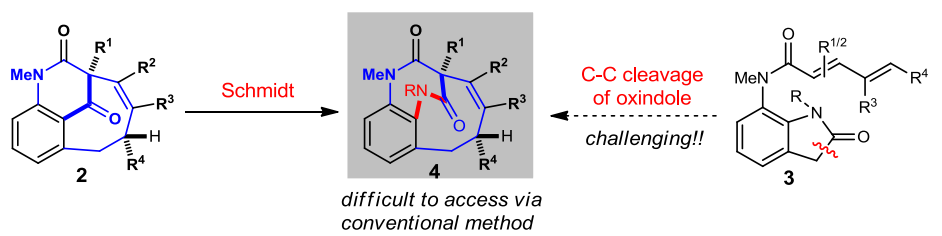

Fig. 4 Derivatization hypothesis. We postulated that a Schmidt reaction of $\mathbf{2}$ maybe able to afford [5.3.2]bridged compound like $\mathbf{4}$, which is very challenging using conventional $\mathrm{C}-\mathrm{C}$ activation method.
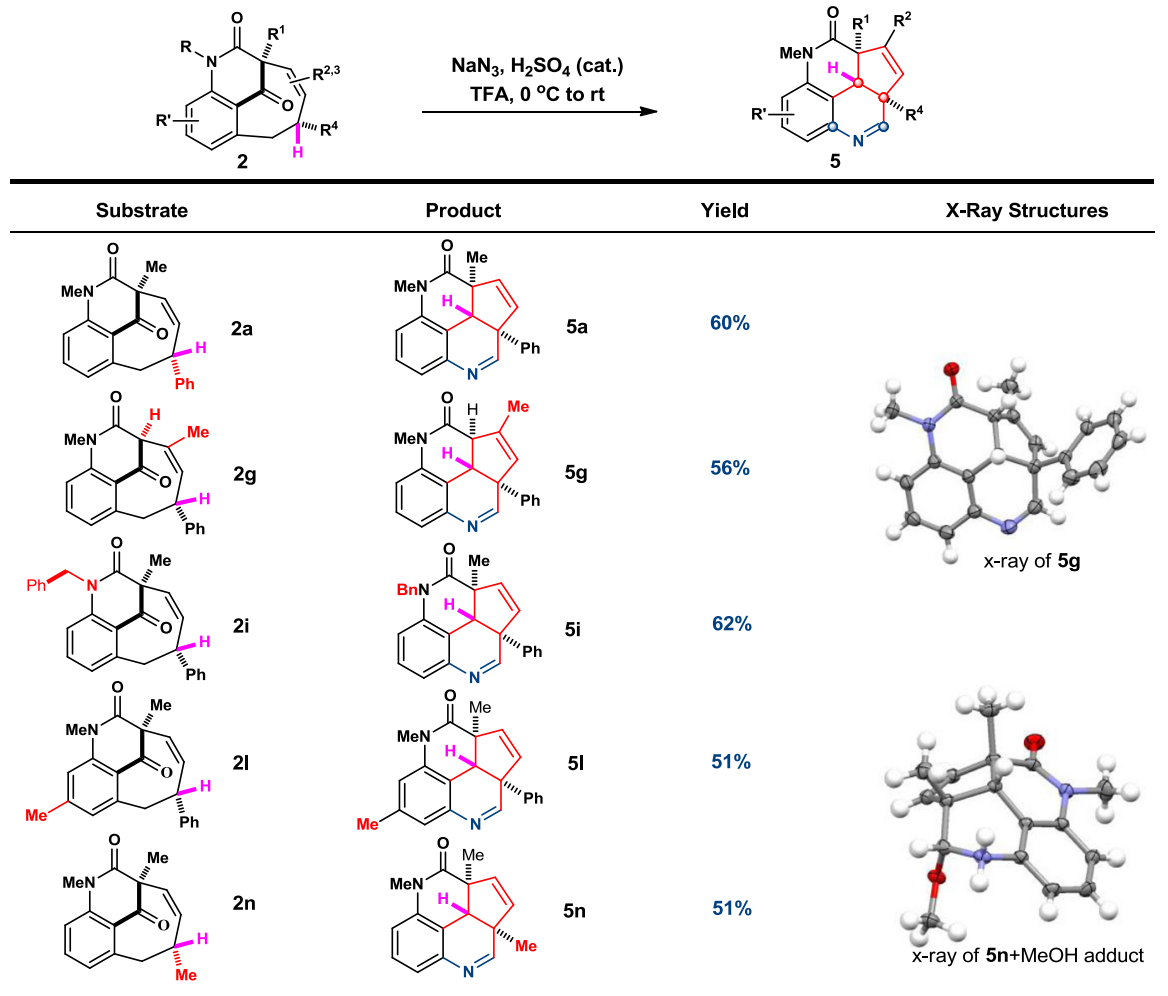

Fig. 5 Cascade rearrangement for fuse-ring system. Conditions: 2 (1.0 equiv.) and $\mathrm{H}_{2} \mathrm{SO}_{4}$ (5 drops) in TFA at $0{ }^{\circ} \mathrm{C}$, then $\mathrm{NaN}$ (3.0 equiv) at rt. $\mathrm{X}$-ray structures was obtained for $\mathbf{5 g}$ and $\mathbf{5 n}+\mathrm{MeOH}$. 


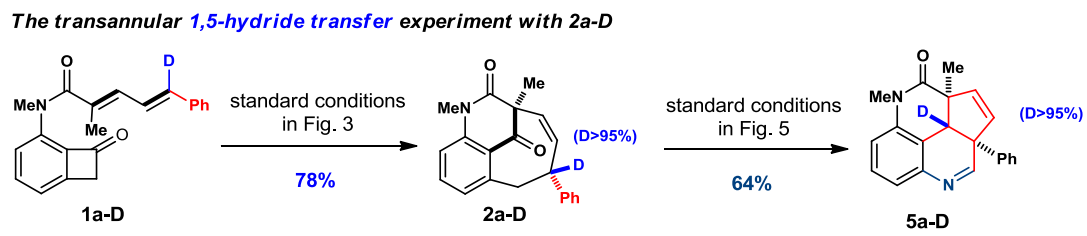

Fig. 6 Mechanism probing of the cascade rearrangement. Standard conditions of $[4+4]$ converted $\mathbf{1 a - D}$ to product $\mathbf{2 a - D}$ diastereoslelctively and with $>95 \%$ Deuterium preserved. Standard conditions of cascade rearrangement afforded $\mathbf{5 a - D}$ with $>95 \%$ Deuterium.

Product 5 consists of a highly congested tetracyclic fused ring cycle rather than the designed simple Schmidt reaction in scheme 1. The mechanism of this transformation was probed using deuterated product $\mathbf{2 a - D}$ as substrate, which was synthesized from correspond 1a-D in 78\% isolated yield using our standard $[4+4]$ conditions (see SI for detail). The fused tetracyclic product 5a-D was successfully obtained in $64 \%$ yield indicating an unambiguous transannular 1,5-deuteryde transfer involved in the cascade skeleton rearrangement (Fig. 6). The dihydroquinoline moiety is a strong evidence of a cation-induced Schmidt rearrangement with $\mathrm{NaN}_{3}$ presumably after the hydride shift (detailed mechanism is provided vide infra).

Based on the previous report and the deuterium experiment, we tentatively proposed the catalytic cycle of the Rh-catalyzed [4 +4 ] annulation between BCBs and pedant dienamides, together with the mechanism of the cascade skeleton rearrangement to form fused tetracycles from bridged bicyclo[5.3.1] undecane (Fig. 7, upper cycle). The $\mathrm{C}_{1}-\mathrm{C}_{8}$ oxidative addition with $\mathrm{Rh}^{\mathrm{I}}$ complex took place first regioselectively, and the equilibration from II to the $\mathrm{C}_{1}-\mathrm{C}_{2}$ cleaved rhodaindanone (green) was competitively overrode by the regioselective migratory insertion into $\gamma, \delta$-olefin due presumably to readily coordination with Rh-center. The resulting penta/hexa coordinated intermediate II was proposed to undergo a migratory insertion with the dienamide, yielding intermediate III. The $\mathrm{Rh}^{\mathrm{III}}$ complex III will undergo reductive elimination thanks to the excess $\mathrm{PPh}_{3}$ ligand, providing the benzofused aza-bicyclo[5.3.1] undecandienone 2 .

To gain insight for our hypothesis of alkyl $\mathrm{C}_{8}$-Rh undergoing migratory insertion rather than acyl $\mathrm{C}_{1}$-Rh into the distal olefin, compound 6 was designed, prepared, and subjected to the standard $[4+4]$ conditions. A formal $[4+2-1]$ annulated product 7 was obtained in $76 \%$ yield (Fig. 8). The formation of product 7 indicated that $\mathrm{C}_{8}$ - $\mathrm{Rh}$ migratory inserted into the terminal olefins first, followed by decarbonylation and reductive elimination. Otherwise, the decarbonylation could not be realized if acyl $\mathrm{C}_{1^{-}}$Rh migrated first.

In the cascade reaction (Fig. 7, lower cycle), the bridged carbonyl group activated by catalytic $\mathrm{H}_{2} \mathrm{SO}_{4}$ will initiated a through space 1,5-hydride transfer followed by a quick E1-type elimination from IV, affording hydride (pink) shifted intermediate $\mathbf{V}$. The benzyl alcohol $\mathbf{V}$ will be protonated by the acid generating benzylic cation, which will induce a Prins-type cyclization obtaining VI. The intermediate VI will be trapped by $\mathrm{NaN}_{3}$ and trigger a Schmidt rearrangement yielding the final polyfused hydroquinoline-containing tetracycle 5.

In summary, we have discovered a Rh-catalyzed type-II $[4+4]$ annulation between $\mathrm{BCBs}$ and pedant dienamide constituting the first synthesis of the anti-Bredt bridgehead benzofused aza-bicyclo[5.3.1] ring system. The methodology featured low catalyst loading $(2.5 \mathrm{~mol} \%)$ and neutral reaction condition with broad substrate scope (23 examples) and high efficiency (up to $89 \%$ yield). The mechanism probing experiment supported that an unusual alkyl $\mathrm{C}_{8}-\mathrm{Rh}$ bond undergoes migratory insertion into distal olefin first. To further diversify the anti-Bredt bridged ring systems, a cascade transformation consisting of transannular

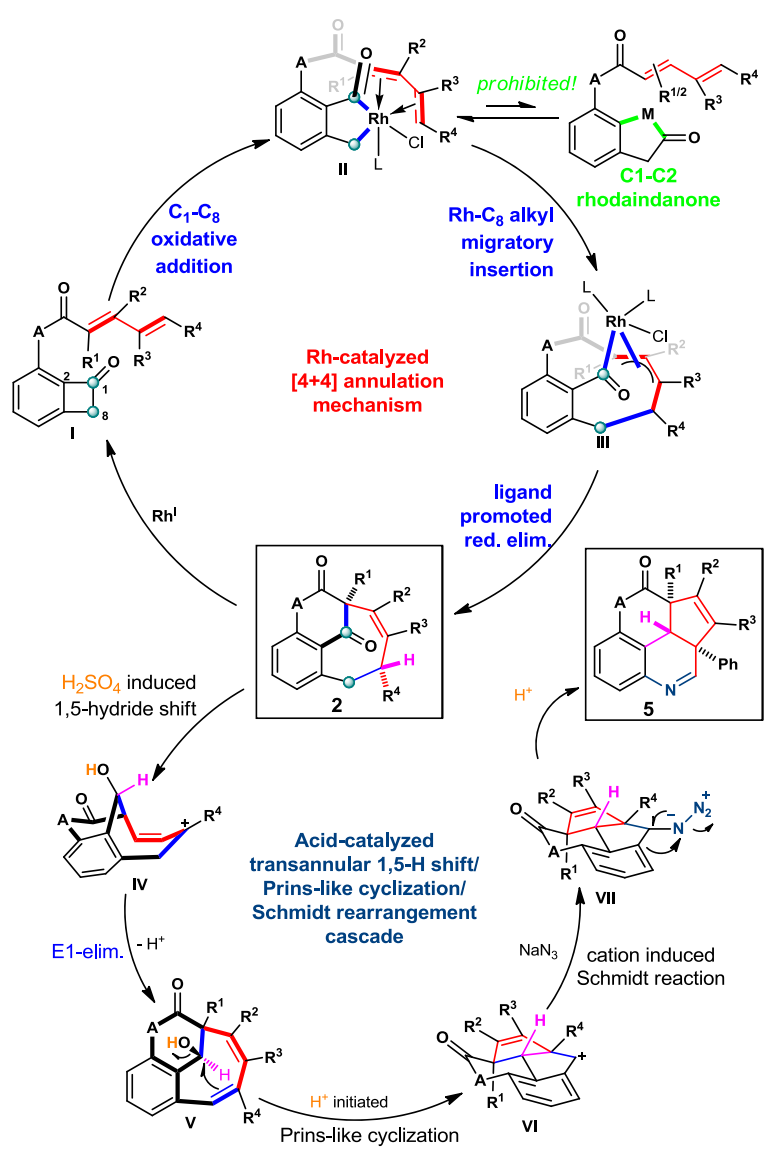

Fig. 7 Proposed mechanism. The upper catalytic cycle is the proposed mechanism for Rh-catalyzed type-II [4+4] cycloaddition, while the lower is the proposed mechanism for acid-catalyzed cascade rearrangement.
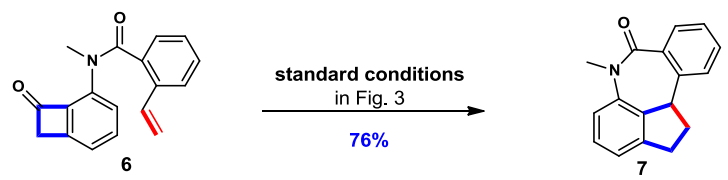

Fig. 8 Control experiments. Substrate 6 was used to probe the mechanism of the migratory insertion step.

1,5-hydride shift, E1 elimination, Prins-like cyclization, and Schmidt rearrangement was discovered, generating polyfused tetracyclic ring systems. A deuterated experiment supported the 1,5-hydride transfer mechanism.

\section{Methods}

Procedure for rh-catalyzed type-II [4+4] annulation to make 2a. A $4 \mathrm{ml}$ oven-dried vial was transferred in a $\mathrm{N}_{2}$-filled glove box and was charged with 1a (40 mg, $0.126 \mathrm{mmol}),\left[\mathrm{Rh}(\mathrm{nbd})_{2} \mathrm{Cl}\right]_{2}(1.5 \mathrm{mg}, 3.15 \mu \mathrm{mol}), \mathrm{PPh}_{3}(4.0 \mathrm{mg}, 0.015$ 


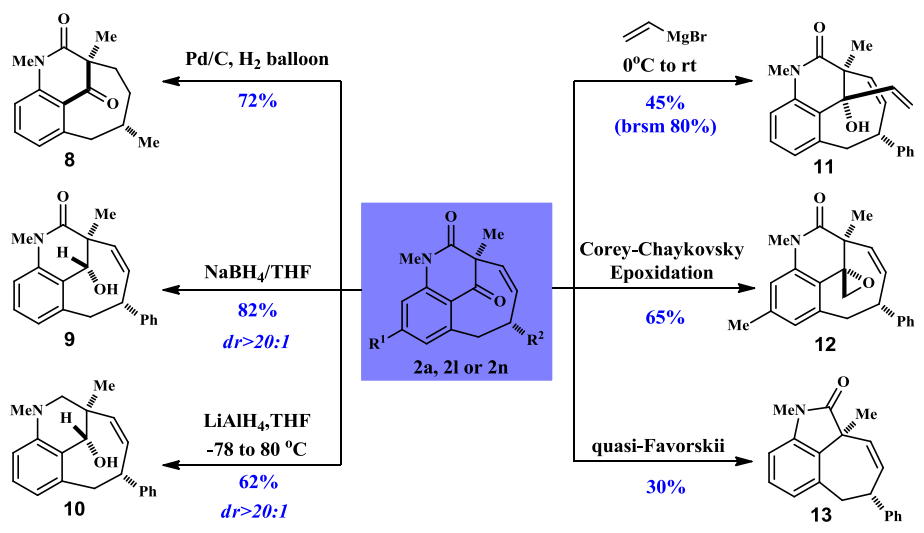

Fig. 9 Applications of compound 2. Hydrogentation with Pd/C afforded 8. Selective reduction can be achieved chemoselectively obtaining 9 and 10. A 1,2addition product $\mathbf{1 1}$ can be diastereoselectively obtained with vinylMgBr. Corey-Chaykovsky expoxidation provide $\mathbf{1 2}$ and quasi-Favorskii led to unexpected fused product 13.

$\mathrm{mmol})$, and 1,4-dioxane $(3.7 \mathrm{ml})$ and the vial was capped. The vial was stirred at $150{ }^{\circ} \mathrm{C}$ for $96 \mathrm{~h}$. Upon completion, it was cooled to room temperature. The solvent was removed by rotavap under reduced pressure and the residue was purified by flash chromatography to afford desired product $\mathbf{2 a}(28.8 \mathrm{mg})$ in $72 \%$ yield and partial 1a $(4.9 \mathrm{mg})$ was recovered. The benzofused bicyclo[5.3.1] products can be further applied in a number of transformations (Fig. 9), Chemoselective reduction can be achieved by using different reagents, e.g., olefin could be hydrogenated obtaining saturated backbone $\mathbf{8}$ in $72 \%$ yield. Ketone and amide functional groups could be reduced to alcohol ( 9 in $82 \%$ yield as a single isomer) and methylene (10 in $60 \%$ yield), respectively, with different hydride reagents. In addition, product $2 \mathrm{a}$ could underwent nucleophilic attack with vinyl Grignard reagent yielding $\mathbf{1 1}$ as a single diastereoisomer ( $45 \%$ yield). When a methylene sulfur ylide was used, an expoxidation product will be isolated in $65 \%$ from 2 l. A quasi-Favorskii reaction can be performed using aqueous $\mathrm{NaOH}$, the fused tricycle product 13 was afforded in $30 \%$ yield.

\section{Data availability}

The data that support the findings of this study are available within the paper and its supplementary information files. Raw data are available from the corresponding author on reasonable request. Materials and methods, experimental procedures, characterization data, 1H, 13C, 19F NMR spectra, and mass spectrometry data are available in the Supplementary Information. The X-ray crystallographic coordinates for structures reported in this study have been deposited at the Cambridge Crystallographic Data Centre (CCDC), under deposition numbers CCDC 1873018 (2a), 1873019 (2b), 1873021 (2j), and $1873022(\mathbf{2 n})$. These data can be obtained free of charge from The Cambridge Crystallographic Data Centre via www.ccdc.cam.ac.uk/data_request/cif.

Received: 25 January 2021; Accepted: 26 April 2021;

Published online: 21 May 2021

\section{References}

1. Mak, J. Y. W., Pouwer, R. H. \& Williams, C. M. Natural products with antiBredt and bridgehead double bonds. Angew. Chem. Int. Ed. 53, 13664-13688 (2014).

2. Krenske, E. H. \& Williams, C. M. Do anti-Bredt natural products exist? Olefin strain energy as a predictor of isolability. Angew. Chem. Int. Ed. 54, 10608-10612 (2015).

3. Maier, W. F. \& Schleyer, P. V. R. Evaluation and prediction of the stability of bridgehead olefins. J. Am. Chem. Soc. 103, 1891-1900 (1981).

4. Tashiro, M. \& Ymato, T. A novel reaction of 8,16-bis(bromomethyl)[2.2] metacyclophanes with phenyl lithium and preparation of 8,16-unsymmetrically disubstituted [2.2]metacyclophanes. Chem. Lett. 11, 61-64 (1982).

5. Tashiro, M. \& Ymato, T. Metacyclophane and related compounds. Part 11. Reaction of 8,16-bis(bromomethyl)[2.2]metacyclophane and 8,16-bis (bromomethy1)-5,13-di-t-butyl[2.2]metacyclophane with Grignard reagents J. Chem. Soc. Perkin. Trans. I, 2165-2171 (1984).

6. Appendino, G., Gariboldi, P. \& Nano, G. M. Crispolide, an unusual hydroperoxysesquiterpene lactone from Tanacetum vulgare. Phytochemistry 21, 1099-1102 (1982).

7. Bohlmann, F. et al. Two sesquiterpene lactones with an additional propiolactone ring from Disynaphia halimifolia. Phytochemistry 20, 1077-1080 (1981).
8. Tempesta, M. S. et al. A new class of sesterterpenoids from the secretion of ceroplastes rubens (Coccidae). J. Chem. Soc., Chem. Commun. 1182-1183 (1983)

9. Paquette, L. A., Hormuth, S. \& Lovely, C. J. Studies directed toward the total synthesis of Cerorubenic Acid-III. 4. Exploration of an organometallic approach to construction of the eastern sector. J. Org. Chem. 60, 4813-4821 (1995).

10. Liu, X. et al. Asymmetric total synthesis of cerorubenic acid-III. J. Am. Chem Soc. 141, 2872-2877 (2019).

11. Zhao, W. Y. Novel syntheses of bridge-containing organic compounds. Chem. Rev. 110, 1706-1745 (2010).

12. Ruiz, M. et al. Domino reactions for the synthesis of bridged bicyclic frameworks: fast access to bicyclo[n.3.1]alkanes. Chem. Soc. Rev. 40, 3445-3454 (2011).

13. Liu, J. et al. Total synthesis of natural products containing a bridgehead double bond. Chem 6, 579-615 (2020).

14. Min, L., Liu, X. \& Li, C.-C. Total synthesis of natural products with bridged Bicyclo[m.n.1] ring systems via type II $[5+2]$ cycloaddition. Acc. Chem. Res. 53, 703-718 (2020).

15. Bear, B. R., Sparks, S. M. \& Shea, K. J. The type 2 intramolecular diels-alder reaction: synthesis and chemistry of bridgehead alkenes. Angew. Chem. Int. Ed. 40, 820-849 (2001).

16. Shea, K. J. et al. Applications of the intramolecular Diels-Alder reaction to the formation of strained molecules. Synthesis of bridgehead alkenes. J. Am. Chem. Soc. 104, 5708-5715 (1982).

17. Wender, P. A. \& Snapper, M. L. Intramolecular nickel catalyzed cycloadditions of bis-dienes: 3 approaches to the taxane skeleton. Tetrahedron Lett. 28, 2221-2224 (1987).

18. Wender, P. A. \& Tebbe, M. J. Nickel(0)-catalyzed intramolecular $[4+4]$ cycloadditions: 5. The type II reaction in the synthesis of Bicyclo[5.3.1] Undecadienes. Synthesis 1991, 1089-1094 (1991).

19. Kennedy, C. R. et al. Regio- and diastereoselective iron-catalyzed $[4+4]$ cycloaddition of 1,3-dienes. J. Am. Chem. Soc. 141, 8557-8573 (2019).

20. Bredt, J. Steric hindrance in the bridge ring (Bredt's rule) and the meso-transposition in condensed ring systems of the hexamethylenes. Justus Liebigs Ann. Chem. 437, 1-13 (1924).

21. Warner, P. M. Strained bridgehead double-bonds. Chem. Rev. 89, 1067-1093 (1989).

22. Jones, W. D. The fall of the C-C bond. Nature 364, 676-677 (1993)

23. Murakami, M. \& Ito, Y. Cleavage of Carbon-Carbon Single Bonds by Transition Metals. Top. Organomet. Chem. 3, 97-129 (1999).

24. Rybtchinski, B. \& Milstein, D. Metal insertion into C-C bonds in solution. Angew. Chem. Int. Ed. 38, 870-883 (1999).

25. Necas, D. \& Kotora, M. Rhodium-catalyzed C-C bond cleavage reactions. Curr. Org. Chem. 11, 1566-1591 (2007).

26. Ruhland, $\mathrm{K}$. Transition-metal-mediated cleavage and activation of $\mathrm{C}-\mathrm{C}$ single bonds. Eur. J. Org. Chem. 2683-2706 (2012).

27. Dong, G. C-C bond activation. (Springer, 2014)

28. Souillart, L. \& Cramer, N. Catalytic C-C Bond Activations via Oxidative Addition to Transition Metals. Chem. Rev. 115, 9410-9464 (2015).

29. Murakami, M. \& Ishida, N. Cleavage of Carbon-Carbon Single Bonds by Transition Metals. (Wiley-VCH, 2015).

30. Fumagalli, G., Stanton, S. \& Bower, J. F. Recent Methodologies that exploit $\mathrm{C}-\mathrm{C}$ single-bond cleavage of strained ring systems by transition metal complexes. Chem. Rev. 117, 9404-9432 (2017). 
31. Chen, P.-H. et al. "Cut and Sew" Transformations via Transition-MetalCatalyzed Carbon-Carbon Bond Activation. ACS Catal. 7, 1340-1360 (2017).

32. Deng, L. \& Dong, G. Carbon-Carbon bond activation of ketones. Trends in Chemistry 2, 183-198 (2020).

33. Murakami, M. \& Ishida, N. Cleavage of Carbon-Carbon $\sigma$-Bonds of FourMembered Rings. Chem. Rev. 121, 264-299 (2021)

34. South, M. S. \& Liebeskind, L. S. Regiospecific Total Synthesis of (士)-Nanaomycin A Using Phthaloylcobalt Complexe. J. Am. Chem. Soc. 106, 4181-4185 (1984).

35. Xu, T. \& Dong, G. Rhodium-Catalyzed Regioselective Carboacylation of Olefins: A C-C Bond Activation Approach for Accessing Fused-Ring Systems. Angew. Chem., Int. Ed. 51, 7567-7571 (2012).

36. $\mathrm{Xu}, \mathrm{T}$. et al. Highly Enantioselective Rh-Catalyzed Carboacylation of Olefins: Efficient Syntheses of Chiral Poly-Fused Rings. J. Am. Chem. Soc. 134, 20005-20008 (2012).

37. Julià-Hernández, F. et al. Nickel-Catalyzed Chemo-, Regio- and Diastereoselective Bond-Formation through Proximal C-C Cleavage of Benzocyclobutenones. Angew. Chem. Int. Ed. 54, 9537-9541 (2015).

38. Ko, H. M. \& Dong, G. Cooperative Activation of Cyclobutanones and Olefins Leads to Bridged Ring Systems by a Catalytic $[4+2]$ Coupling. Nat. Chem. 6 , 739-744 (2014).

39. Souillart, L., Parker, E. \& Cramer, N. Highly Enantioselective Rhodium(I)Catalyzed Activation of Enantiotopic Cyclobutanone C-C Bonds. Angew. Chem., Int. Ed. 53, 3001-3005 (2014).

40. Souillart, L. \& Cramer, N. Highly Enantioselective Rhodium(I)-Catalyzed Carbonyl Carboacylations Initiated by C-C Bond Activation. Angew. Chem., Int. Ed. 53, 9640-9644 (2014).

41. Zhou, X. \& Dong, G. $(4+1)$ vs $(4+2)$ : Catalytic Intramolecular Coupling between Cyclobutanones and Trisubstituted Allenes via C-C Activation. J. Am. Chem. Soc. 137, 13715-13721 (2015).

42. Liu, L., Ishida, N. \& Murakami, M. Atom- and Step-Economical Pathway to Chiral Benzobicyclo[2.2.2] octenones through Carbon-Carbon Bond Cleavage. Angew. Chem., Int. Ed. 51, 2485-2488 (2012).

43. Hou, S.-H. et al. Enantioselective Type II Cycloaddition of Alkynes via C-C Activation of Cyclobutanones: Rapid and Asymmetric Construction of [3.3.1] Bridged Bicycles. J. Am. Chem. Soc. 142, 13180-13189 (2020).

44. Lu, G. et al. Computational Study of Rh-Catalyzed Carboacylation of Olefins: Ligand-Promoted Rhodacycle Isomerization Enables Regioselective C-C Bond Functionalization of Benzocyclobutenones. J. Am. Chem. Soc. 137, 8274-8283 (2015).

45. Qiu, B. et al. Catalytic enantioselective synthesis of 3, 4-polyfused oxindoles with quaternary all-carbon stereocenters: a Rh-catalyzed C-C activation approach. Org. Lett. 20, 7689-7693 (2018).

46. Yu, Z.-X., Wang, Y. \& Wang, Y. Transition-Metal-Catalyzed Cycloadditions for the Synthesis of Eight-Membered Carbocycles. Chem. Asian J. 5, 1072-1088 (2010).

47. Wang, Y. \& Yu, Z.-X. Rhodium-Catalyzed [5+2+1] Cycloaddition of Ene-Vinylcyclopropanes and CO: Reaction Design, Development, Application in Natural Product Synthesis, and Inspiration for Developing New Reactions for Synthesis of Eight-Membered Carbocycles. Acc. Chem. Res. 48, 2288-2296 (2015).

48. Brown, T. L. \& Lee, K. J. Ligand steric properties. Coord. Chem. Rev. 128, 89-116 (1993).

49. Tambar, U. K. \& Stoltz, B. M. The direct acyl-alkylation of arynes. J. Am Chem. Soc. 127, 5340-5341 (2005).

50. Zhu, C. et al. Enantioselective palladium-catalyzed intramolecular a-arylative desymmetrization of 1,3-diketones. J. Am. Chem. Soc. 139, 16486-16489 (2017).
51. Wang, M. et al. Radical-mediated C-C cleavage of unstrained cycloketones and DFT study for unusual regioselectivity. Nat. Commun. 11, 672 (2020)

52. Fu, X.-F., Xiang, Y. \& Yu, Z.-X. RhI-Catalyzed Benzo/[7+1] Cycloaddition of Cyclopropyl-Benzocyclobutenes and $\mathrm{CO}$ by Merging Thermal and MetalCatalyzed C-C Bond Cleavages. Chem. Eur. J. 21, 4242-4246 (2015).

53. Wang, L.-N. \& Yu, Z.-X. Transition-Metal-Catalyzed Cycloadditions for the Synthesis of Eight-Membered Carbocycles: An Update from 2010 to 2020. Chin. J. Org. Chem. 40, 3536-3558 (2020)

\section{Acknowledgements}

The project was supported by the Fundamental Research Funds for the Central Universities (202041003), NSFC (Nos. 81991522, U1706213, and 81973232) and Shandong Science Fund for Distinguished Young Scholars (ZR2020JQ32) for financial support. The projected was partially funded by National Science and Technology Major Project of China (No. 2018ZX09735-004), Youth Innovation Plan of Shandong Province (2019KJM004) and Jinan municipal government fund (2019GXRC039). T.X. is a Taishan Youth Scholar (tsqn20161012). GBD of U. Chicago is acknowledged for helpful discussions.

\section{Author contributions}

T.X. conceived the project and wrote the manuscript. J.Z. performed all the key reactions, collected data, and prepared SI. X.W. helped preparing BCB and collecting data

\section{Competing interests}

The authors declare no competing interests.

\section{Additional information}

Supplementary information The online version contains supplementary material available at https://doi.org/10.1038/s41467-021-23344-0.

Correspondence and requests for materials should be addressed to T.X.

Peer review information Nature Communications thanks the anonymous reviewer(s) for their contribution to the peer review of this work.

Reprints and permission information is available at http://www.nature.com/reprints

Publisher's note Springer Nature remains neutral with regard to jurisdictional claims in published maps and institutional affiliations.

Open Access This article is licensed under a Creative Commons Attribution 4.0 International License, which permits use, sharing, adaptation, distribution and reproduction in any medium or format, as long as you give appropriate credit to the original author(s) and the source, provide a link to the Creative Commons license, and indicate if changes were made. The images or other third party material in this article are included in the article's Creative Commons license, unless indicated otherwise in a credit line to the material. If material is not included in the article's Creative Commons license and your intended use is not permitted by statutory regulation or exceeds the permitted use, you will need to obtain permission directly from the copyright holder. To view a copy of this license, visit http://creativecommons.org/ licenses/by/4.0/

(C) The Author(s) 2021 